\title{
Prehospital Modified HEART Score Predictive of 30-Day Adverse Cardiac Events
}

\author{
Jason P. Stopyra, MD $;^{1}$ William S. Harper, MD $;{ }^{1}$ Tyson J. Higgins, MD ${ }^{2}$ Julia V. Prokesova, MS, IV; ${ }^{1}$ \\ James E. Winslow, MD, MPH; ${ }^{1}$ Robert D. Nelson, MD; ${ }^{1}$ Roy L. Alson, MD, PhD; ${ }^{1}$ Christopher A. \\ Davis, MD; ${ }^{1}$ Gregory B. Russell, MS; ${ }^{1}$ Chadwick D. Miller, MD, MS; ${ }^{1}$ Simon A. Mahler, MD, MS ${ }^{1}$
}

1. Wake Forest School of Medicine, Winston-Salem, North Carolina USA

2. John Peter Smith Hospital, Fort Worth, Texas USA

\section{Correspondence:}

Jason P. Stopyra, MD

Department of Emergency Medicine

Wake Forest School of Medicine

Medical Center Boulevard

Winston-Salem, North Carolina 27157 USA

E-mail: jstopyra@wakehealth.edu

Conflicts of interest/funding: This study was funded by the American Heart Association (Dallas, Texas USA; 12CRP12000001).

Dr. Mahler receives funding from the Donaghue Foundation (West Hartford, Connecticut USA), Duke Endowment (Charlotte, North Carolina USA), Abbott Point of Care (Princeton, New Jersey USA), Roche Diagnostics (Indianapolis, Indiana USA), and the National Heart, Lung, and Blood Institute (NHLBI; Bethesda, Maryland USA; 1 R01 HL118263-01, L30 HL120008). Dr. Mahler is the chief medical officer for Decision Point Informatics (Raleigh, North Carolina USA). Dr. Stopyra receives funding from Abbott Point of Care.

Keywords: acute coronary syndrome; chest pain; decision support techniques; Emergency Medical Services

\section{Abbreviations:}

ACS: acute coronary syndrome

AMI: acute myocardial infarction

ECG: electrocardiogram

ED: emergency department

EMS: Emergency Medical Services

HEAR: History, ECG, Age, Risk Factors

Abstract

Introduction: The History, Electrocardiogram (ECG), Age, Risk Factors, and Troponin (HEART) score is a decision aid designed to risk stratify emergency department (ED) patients with acute chest pain. It has been validated for ED use, but it has yet to be evaluated in a prehospital setting.

Hypothesis: A prehospital modified HEART score can predict major adverse cardiac events (MACE) among undifferentiated chest pain patients transported to the ED.

Methods: A retrospective cohort study of patients with chest pain transported by two county-based Emergency Medical Service (EMS) agencies to a tertiary care center was conducted. Adults without ST-elevation myocardial infarction (STEMI) were included. Inter-facility transfers and those without a prehospital 12-lead ECG or an ED troponin measurement were excluded. Modified HEART scores were calculated by study investigators using a standardized data collection tool for each patient. All MACE (death, myocardial infarction $[\mathrm{MI}]$, or coronary revascularization) were determined by record review at 30 days. The sensitivity and negative predictive values (NPVs) for MACE at 30 days were calculated.

Results: Over the study period, 794 patients met inclusion criteria. A MACE at 30 days was present in $10.7 \%(85 / 794)$ of patients with 12 deaths (1.5\%), $66 \mathrm{MIs}(8.3 \%)$, and 12 coronary revascularizations without MI (1.5\%). The modified HEART score identified $33.2 \%(264 / 794)$ of patients as low risk. Among low-risk patients, $1.9 \%(5 / 264)$ had MACE (two MIs and three revascularizations without MI). The sensitivity and NPV for 30-day MACE was 94.1\% (95\% CI, 86.8-98.1) and 98.1\% (95\% CI, 95.6-99.4), respectively.

Conclusions: Prehospital modified HEART scores have a high NPV for MACE at 30 days. A study in which prehospital providers prospectively apply this decision aid is warranted.

Stopyra JP, Harper WS, Higgins TJ, Prokesova JV, Winslow JE, Nelson RD, Alson RL, Davis CA, Russell GB, Miller CD, Mahler SA. Prehospital modified HEART score predictive of 30-day adverse cardiac events. Prehosp Disaster Med. 2018;33(1):58-62.

\section{Introduction}

Utilization of Emergency Medical Services (EMS) is on the rise, as is the number of patients calling for assistance due to the complaint of chest pain. ${ }^{1-3}$ Chest pain is second (11.0\%) only to minor injuries as the most common call for help received by public service answer points, ${ }^{4}$ and it accounts for $20.0 \%$ of calls that are deemed the highest priority. ${ }^{5}$ In the 1980 s, $28.0 \%$ of all prehospital patients had acute myocardial infarction (AMI), but this number decreased to $12.0 \%$ in 2010, indicating greater use of EMS by low-risk patients. ${ }^{6}$

\author{
HEART: History, ECG, Age, Risk Factors, and \\ Troponin \\ MACE: major adverse cardiac events \\ MI: myocardial infarction \\ NPV: negative predictive value \\ POC: point-of-care \\ STEMI: ST-elevation myocardial infarction
}

Received: February 10, 2017

Revised: May 22, 2017

Accepted: June 23, 2017

Online publication: January 10, 2018

doi:10.1017/S1049023X17007154 
As prehospital health care evolves, paramedics continue to expand their scope of practice and make more complex medical decisions. For example, the prehospital health care environment has seen great success using decision support tools for stroke evaluation. Studies call for a tool that can be used in the prehospital environment to determine whether patients with acute chest pain are at low or high risk for acute coronary syndrome (ACS).,7 A systematic review identified increasing age, male gender, elevated heart rate, low systolic blood pressure, and ST-elevation myocardial infarction (STEMI) or depression on 12-lead electrocardiogram (ECG) as prehospital factors associated with acute life-threatening conditions in non-traumatic chest pain patients. ${ }^{6}$ However, to date, decision aids haven't been deployed to help paramedics risk stratify patients with acute chest pain who are without evidence of STEMI on ECG.

The History, ECG, Age, Risk Factors, and Troponin (HEART) score is a validated decision aid that was developed to help risk stratify patients with chest pain in the emergency department (ED) setting. ${ }^{8,9}$ This study hypothesizes that paramedics using a modified HEART score will be able to accurately risk stratify patients with chest pain. The objective of this study was to determine the sensitivity and specificity of modified and traditional HEART scores, calculated based on paramedic's prehospital reports for 30-day major adverse cardiac events (MACE). Furthermore, this study was designed to determine whether a prospective study of paramedics using a HEART score and pointof-care (POC) troponin measurements is warranted.

\section{Methods \\ Study Design}

This is a retrospective cohort study of patients with undifferentiated chest pain transported by Surry County (North Carolina USA) EMS (from November 2013 through December 2014) and Forsyth County (Georgia USA) EMS (from November 2013 through June 2014) to Wake Forest Baptist Health Medical Center (Winston-Salem, North Carolina USA) ED. The study was approved by the Internal Review Board at Wake Forest Baptist Health.

\section{Study Setting and Population}

The study institution is a tertiary academic referral center with an annual ED volume of approximately 104,000 patients per year. Surry County is a rural county with a population of 73,000 that responds to 26,000 calls per year. Forsyth County is an urban county with a population of 361,000 that responds to 61,000 calls per year. The EMS assessment protocols require identification and documentation of patient's past medical history and history of present illness as it pertains to the patient's complaint of chest pain. A 12-lead ECG is indicated by the Chest Pain EMS Protocol and was required for study inclusion. Patients $\geq 18$ years old with a chief complaint of chest pain transported via ambulance and without evidence of STEMI on ECG were included. Interfacility transfers and those without a prehospital 12-lead ECG or an in-hospital troponin measurement were excluded. The decision to transport to Wake Forest Baptist Medical Center was at the discretion of the paramedic provider or patient preference.

\section{Study Protocol and Measures}

Patients underwent a blinded chart review conducted by study investigators (WSH, TJH, and JVP). Each investigator received data collection training specific to the EMS' prehospital electronic

\begin{tabular}{|l|c|}
\hline History & Value \\
\hline Highly Suspicious & 2 \\
\hline Moderately Suspicious & 1 \\
\hline Slightly Suspicious & 0 \\
\hline ECG & 2 \\
\hline Significant ST-Depression & 1 \\
\hline Non-Specific Repolarization Disturbance & 0 \\
\hline Normal & \\
\hline Age & 2 \\
\hline$\geq 65$ years & 1 \\
\hline $45-65$ years & 0 \\
\hline$\leq 45$ years & 2 \\
\hline Risk Factors & 1 \\
\hline$\geq 3$ Risk Factors or History of Atherosclerotic Disease & 0 \\
\hline 1 or 2 Risk Factors & $\mathbf{0 - 8}$ \\
\hline No Risk Factors Known & \\
\hline Total & \\
\hline $\begin{array}{l}\text { "Low Risk" = 0-3 and Normal Troponin } \\
\text { "High Risk" = 4-8 or Troponin (>0.065 ng/ml) }\end{array}$ \\
\hline
\end{tabular}

Figure 1. Modified HEART Score for Chest Pain Patients. Abbreviations: ECG, electrocardiogram; HEART, History, ECG, Age, Risk Factors, and Troponin.

health record (ESO Solutions; Austin, Texas USA). For data abstraction, they utilized a standardized data collection tool with well-defined variables. Keyword analysis of the document health record was used to objectively determine if the history was highly, moderately, or slightly suspicious for ACS. Low-risk descriptors included: reproducible, positional, sharp, and pinpoint. Left or center location and heavy, pressure, tight, sharp, exertional, improvement with nitroglycerin, nausea, vomit, diaphoresis, and radiation to arm or jaw were high-risk descriptors. If the patient had mostly low-risk descriptors (at least two more low-risk words than high-risk words), the history was considered slightly suspicious. If they had mostly high-risk descriptors (at least two more high-risk words than low-risk words), the history was considered highly suspicious. If they had an equal or nearly equal mixture (only one additional high- or low-risk descriptor), the history was considered moderately suspicious. Monitoring was accomplished by abstractors spot checking each other's chart reviews. Any disagreements were settled by consensus between the two, or the involvement of the principle investigator. The paramedics' documentation of their 12-lead ECG interpretation was collected. Patient age and risk factors (hypertension, diabetes, smoking, and hypercholesterolemia) were obtained and then the H-E-A-R components of a score were calculated. The initial contemporary troponin measure from the ED was used to complete the $\mathrm{T}$ component of the HEART score (Figure 1). Periodic meetings were held with the abstractors and principle investigator to review the chart review methods. ${ }^{10}$

The HEART score is a clinical decision aid that risk stratifies patients into a low-risk or high-risk group for ACS. ${ }^{8,9,11-13}$ In this analysis, a modified version of the HEART score was tested by removing the "Troponin" component, creating a History, ECG, Age, Risk Factors (HEAR) score and assessing the first troponin measure independently. To be considered low risk, a patient must have both a HEAR score of $\leq$ three and a negative initial ED troponin. Unlike the traditional HEART score, this modified version doesn't allow patients with a positive troponin to be classified as low risk. Patients with a positive initial troponin or HEAR score of $\geq$ four were classified as high risk. Since POC troponin testing is not routinely available in the prehospital setting, the performance of a HEAR score without the use of a troponin measure was also tested. 
A MACE was defined as a composite endpoint of all-cause mortality, myocardial infarction (MI), or coronary revascularization during the index visit or within 30 days, as determined by record review at 30 days. Myocardial infarction was determined based on the universal definition of AMI by Thygesen et al using the Siemens cTnI-Ultra assay (Siemens; Munich, Germany) with a $99^{\text {th }}$ percentile URL of $0.040 \mathrm{ng} / \mathrm{mL} \cdot{ }^{14}$ Coronary revascularization was defined as emergent or non-emergent revascularization procedures, including angioplasty with or without stent placement, or coronary artery bypass surgery. All patients received a review of the electronic medical record to determine MACE within 30 days. Inpatients lost to follow-up, the North Carolina Death Index was cross referenced to evaluate for death within 30 days.

\section{Study Analysis}

The primary outcome measure was the presence or absence of MACE during the index visit or within 30 days. To describe the study population, descriptive statistics, including means and standard deviations for continuous data and frequencies, proportions, and $95 \%$ exact binomial confidence intervals, were calculated. The HEAR score, as well as the traditional and modified HEART scores, were calculated from EMS data and initial troponin to classify the patient into a risk group. The percentage of patients identified as low risk; the sensitivity, specificity, and positive and negative predictive values (NPVs); and positive and negative likelihood ratios of the combination of the HEART score and initial troponin for MACE were calculated, along with the corresponding 95\% exact binomial confidence intervals. Patients with incomplete follow up $(5.9 \%$; 47/794) were considered to be free of 30-day MACE events. Odds ratios for MACE were calculated for each version of the HEART score (modified, traditional, and HEAR). Statistical analysis was performed using SAS 9.4 (SAS; Cary, North Carolina USA).

\section{Results}

From November 2013 through December 2014, 794 chest pain patients meeting study inclusion criteria were brought to Wake Forest Baptist Health ED via Surry or Forsyth County ambulances from a scene. Characteristics of the cohort are summarized in Table 1. Complete 30-day follow-up data were available in 94.0\% (747/794) of the patients.

A MACE at 30 days was present in $10.7 \%(85 / 794)$ of patients with 12 deaths (1.5\%), $66 \mathrm{MIs}(8.3 \%)$, and 12 coronary revascularizations without MI (1.5\%). The modified HEART score identified 33.2\% (264/794) of patients as low risk. Among patients with a low-risk assessment, $1.9 \%(5 / 264 ; 95 \% \mathrm{CI}$, 0.6-4.4) had MACE (two MIs and three revascularizations without MI) compared to $15.1 \%$ (80/530; 95\% CI, 12.1-18.4) of high-risk patients (12 deaths, $59 \mathrm{MIs}$, and nine revascularizations without MI). A high-risk assessment by the modified HEART score was associated with an odds ratio for MACE of 9.2 (95\% CI, 3.6-23.0). The sensitivity of the modified HEAR score for 30-day MACE was 94.1\% (95\% CI, 86.8-98.1) with a NPV of $98.1 \%$ (95\% CI, 95.6-99.4). Table 2 shows a summary of the modified HEART score test characteristics. A comparison of the test characteristics for the modified HEART score, traditional HEART score, and HEAR score are summarized in Table 3.

\begin{tabular}{|l|c|}
\hline Patient Characteristics & \\
\hline Age - mean & 57.7 \\
\hline Female & $373(47.0 \%)$ \\
\hline Race & \\
\hline White or Caucasian & $415(52.3 \%)$ \\
\hline Black or African American & $335(42.2 \%)$ \\
\hline Asian & $7(0.9 \%)$ \\
\hline American Indian & $1(0.1 \%)$ \\
\hline Other & $36(4.5 \%)$ \\
\hline Ethnicity & \\
\hline Hispanic or Latino & $35(4.4 \%)$ \\
\hline Symptom Description & \\
\hline Middle-or-Left-Sided Chest Pain & $306(38.5 \%)$ \\
\hline Heavy Chest Pain & $376(47.4 \%)$ \\
\hline Diaphoresis & $62(7.8 \%)$ \\
\hline Radiation & $207(26.1 \%)$ \\
\hline Nausea/Vomiting & $143(18.0 \%)$ \\
\hline Exertional & $59(7.4 \%)$ \\
\hline Relief of Symptoms by Sublingual Nitrates & $320(40.3 \%)$ \\
\hline Risk Factors & $327(41.2 \%)$ \\
\hline Known CAD & $30(3.8 \%)$ \\
\hline Prior Stroke & $7(0.9 \%)$ \\
\hline Peripheral Arterial Disease & \\
\hline Stopyra $\odot 2018$ Prehospital and Disaster Medicine
\end{tabular}

Table 1. Patient Characteristics

Abbreviation: $\mathrm{CAD}$, coronary artery disease.

\section{Discussion}

In this study, the performance of a prehospital modified HEART score (a HEAR score used with an initial ED troponin measure) to risk stratify patients with acute chest pain is reported. This analysis is the first to demonstrate that paramedics routinely obtain the information needed to calculate a HEAR score, and this score can be combined with an initial troponin measure to achieve a high sensitivity and NPV for MACE. Patients with acute chest pain are highly prevalent and pose a challenge to EMS systems without local hospitals that perform interventional cardiology or stress testing on weekends and holidays. A decision aid, such as the HEART score, used to assist prehospital risk stratification could guide prehospital providers to change their destination to facilities that have these capabilities. The identification of patients with high- or low-risk assessments may also assist prehospital providers in making treatment decisions. For example, a low-risk assessment may help prehospital providers avoid nitrates and anticoagulants in low-risk patients. 


\begin{tabular}{|l|c|c|c|}
\hline & \multicolumn{2}{|c|}{ MACE } & \\
\hline HEART Score & Yes (n) & No (n) & Total (n) \\
\hline High Risk (n) & 80 & 450 & 530 \\
\hline Low Risk (n) & 5 & 259 & 264 \\
\hline Total (n) & 85 & 709 & 794 \\
\hline Sensitivity & $94.1 \% ; 95 \%$ Cl, 86.8-98.1 \\
\hline Specificity & $36.5 \% ; 95 \%$ Cl, 33.0-40.2 \\
\hline Positive Predictive Value & $15.1 \% ; 95 \%$ Cl, 12.2-18.4 \\
\hline Negative Predictive Value & \multicolumn{2}{|c|}{$98.1 \% ; 95 \%$ Cl, 95.6-99.4 } \\
\hline Positive Likelihood Ratio & $1.48 \% ; 95 \%$ Cl, 1.36-1.60 \\
\hline Negative Likelihood Ratio & \multicolumn{2}{|c|}{$0.16 \% ; 95 \%$ Cl, 0.02-0.31 } \\
\hline
\end{tabular}

Table 2. Test Characteristics of the Modified HEART Score (HEAR 4+ or Positive Troponin) for Detection of MACE among EMS Patients with Chest Pain

Abbreviations: EMS, Emergency Medical Services; HEART, History, ECG, Age, Risk Factors, and Troponin; MACE, major adverse cardiac events.

The HEART score and related HEART Pathway have been adopted in many EDs to determine which patients are at low risk for MACE and are unlikely to benefit from inpatient or observation unit stress testing. A recent randomized trial showed that, compared to usual care, use of the HEART Pathway increased early discharges by $21.3 \%$, decreased length of stay in the hospital by 12 hours, and decreased objective cardiac testing at 30 days by $12.0 \%$ without increasing adverse events. ${ }^{15,16}$ The use of the HEART Pathway as a decision aid for patients with undifferentiated chest pain has also shown a significant cost savings. ${ }^{17}$ The prehospital arena is a natural extension of this work due to the large number of patients that present to the $\mathrm{ED}$ via ambulance. The ability to start objective risk stratification early in a patient's care pathway may help to better allocate resources more efficiently.

The modified HEART score provided a sensitivity of $94.0 \%$ and a NPV of $98.0 \%$ for MACE in a prehospital cohort of patients with a moderate rate of MACE (10.7\%). The missed MACE rate of $1.9 \%$ in this study is very similar to the missed MACE rate of $1.7 \%$ reported in prior HEART score validations. ${ }^{12,13}$ The sensitivity and NPV of the HEART score would likely improve with training of the prehospital providers and its application prospectively with POC troponins. Prehospital providers have historically been very comfortable using algorithms to guide the care they provide to patients. The HEART score is a good example of a quick, reliable screening tool that could be easily embedded within EMS electronic medical records.

Health care providers are called to provide the right care to the right patient at the right time. The prospective application of the HEART score has the potential to greatly assist prehospital providers in accomplishing this goal. The American health care system will face efficiency benchmarks in the coming years like never before. The transition to value-based care will require health care providers to challenge historic practices with the new goal to safely provide cost-contained care to as many patients as possible. The ability to obtain a prehospital POC troponin would empower an autonomous application of the HEART score risk stratification tool. This lab value has the potential to significantly improve patient flow in the ED. Low-risk patients that need a 3-hour troponin before discharge, or high-risk patients with a negative first troponin that require stress testing, could be quickly moved to an observation bed. Cardiology consultation for patients with a positive troponin could also be streamlined. Prehospital providers may be able to use the modified HEART score to determine what hospital resources are needed to complete their patients' chest pain evaluation to avoid transfers and better match a patient's risk to the available health system resources. For example, paramedics could avoid transporting high-risk patients to community hospitals without cardiac catheterization capabilities, or on weekends and holidays, avoid facilities without the ability to perform stress testing.

An exciting future application of this work that truly moves prehospital services in line with the era of mobile integrated health is the potential to allow low-risk HEART score patients to stay home and then have a return scheduled 3-hour visit by a paramedic to repeat a troponin and ECG. The efficiency and patient convenience of this model would allow the patient to not even leave their home. It goes without being said that it is extremely important that other serious etiologies of chest pain be clinically ruled out through protocol and algorithm for this process to have utility. This process may be alarming for a 911 EMS agency to consider implementing, but with the appropriate evidence to support its use, it may be a safe and efficient way to contain their own costs and wisely allocate resources. More evidence is required to support this kind of endeavor, but a reasonable next step is to determine if POC troponin assays can be reliably obtained in the prehospital environment during ambulance transport.

Given the promising results of this study, it would be wise to proceed with a prospective prehospital analysis of the HEAR score, associated a POC troponin analysis performed prior to ED arrival. Though there are significant administrative hurdles that would have to be overcome for a study such as this to be performed in the United States, it is reasonable to foresee the future utility of this data.

\section{Limitations}

There are several limitations to this study. This retrospective study used patients from two EMS systems that transported patients to a single medical center. The results of this study may not be generalizable to chest pain in other EMS systems or patients presenting to other institutions. A prehospital registry was retrospectively analyzed to determine a patient's HEAR score. This registry was not designed to determine a HEAR score and therefore was not universally complete in the data that it offered the abstractors in this study. The registry did not contain a specific field to determine whether a patients' history was highly, moderately, slightly, or not suggestive of ACS. Body mass index was not available. The primary outcome measure in this study was MACE during the index visit or within 30 days, but 47 patients had no follow-up data following their index visit. This could have resulted in a misclassification bias and under-estimation of the primary outcome. However, none of these patients appeared in the North Carolina Death Index. 


\begin{tabular}{|l|c|c|c|}
\hline & HEAR Score & Traditional HEART & Modified HEART \\
\hline Low Risk \% (95\% Cl) & $40.0 \%(36.6-43.5)$ & $35.3 \%(31.9-38.7)$ & $33.2 \%(30.0-36.6)$ \\
\hline Odds Ratio $(95 \% \mathrm{Cl})$ & $2.7(1.6-4.7)$ & $6.0(2.8-12.6)$ & $9.2(3.6-23.0)$ \\
\hline Sensitivity \% (95\% Cl) & $78.8 \%(68.6-86.9)$ & $90.6 \%(82.2-95.9)$ & $94.1 \%(86.8-98.1)$ \\
\hline Specificity \% (95\% Cl) & $42.3 \%(38.6-46.0)$ & $38.4 \%(34.8-42.1)$ & $36.5 \%(33.0-40.2)$ \\
\hline Positive Predictive Value \% $(95 \% \mathrm{Cl})$ & $14.1 \%(11.1-17.5)$ & $15.0 \%(12.0-18.4)$ & $15.1 \%(12.2-18.4)$ \\
\hline Negative Predictive Value \% (95\% Cl) & $94.3 \%(91.2-96.6)$ & $97.1 \%(94.5-98.8)$ & $98.1 \%(95.6-99.4)$ \\
\hline Positive Likelihood Ratio $(95 \% \mathrm{Cl})$ & $1.37(1.18-1.55)$ & $1.47(1.33-1.61)$ & $1.48(1.36-1.60)$ \\
\hline Negative Likelihood Ratio $(95 \% \mathrm{Cl})$ & $0.50(0.28-0.72)$ & $0.25(0.07-0.42)$ & Stopyra $\odot 2018$ Prehospital and Disaster Medicine \\
\hline
\end{tabular}

Table 3. Comparison of Test Characteristics of the HEAR Score, Traditional and Modified HEART Scores, for Detection of MACE among EMS Patients with Chest Pain Abbreviations: EMS, Emergency Medical Services; HEART, History, ECG, Age, Risk Factors, and Troponin; MACE, major adverse cardiac events.

\section{Conclusions}

Prehospital modified HEART scores have a high NPV for MACE at 30 days. Furthermore, this study demonstrates that prehospital providers currently obtain and document the data required to inform a HEAR score. The use of a prehospital clinical

References

1. Lowthian JA, Cameron PA, Stoelwinder JU, et al. Increasing utilization of emergency ambulances. Aust Health Rev. 2011;35(1):63-69.

2. Pittet V, Burnand B, Yersin B, Carron PN. Trends of pre-hospital Emergency Medical Services activity over 10 years: a population-based registry analysis. $B M C$ Health Serv Res. 2014;14:380.

3. Saddichha S, Saxena MK. Is every chest pain a cardiac event? An audit of patients with chest pain presenting to emergency services in India. Intern Emerg Med. 2009;4(3):235-239.

4. Moller TP, Ersboll AK, Tolstrup JS, et al. Why and when citizens call for emergency help: an observational study of 211,193 medical emergency calls. Scand J Trauma Resusc Emerg Med. 2015;23:88.

5. Burman RA, Zakariassen E, Hunskaar S. Acute chest pain - a prospective population based study of contacts to Norwegian emergency medical communication centers. BMC Emerg Med. 2011;11:9.

6. Wibring K, Herlitz J, Christensson L, Lingman M, Bang A. Prehospital factors associated with an acute life-threatening condition in non-traumatic chest pain patients - a systematic review. Int J Cardiol. 2016;219:373-379.

7. Thang ND, Sundstrom BW, Karlsson T, Herlitz J, Karlson BW. ECG signs of acute myocardial ischemia in the prehospital setting of a suspected acute coronary syndrome and its association with outcomes. Am J Emerg Med. 2014;32(6):601-605.

8. Six AJ, Backus BE, Kelder JC. Chest pain in the emergency room: value of the HEART score. Neth Heart J. 2008;16(6):191-196.

9. Backus BE, Six AJ, Kelder JC, et al. Chest pain in the emergency room: a multicenter validation of the HEART score. Crit Pathw Cardiol. 2010;9(3):164-169. decision aid combined with a troponin measure has the potential to significantly improve the quality and efficiency of the evaluation and treatment of patients with chest pain. The data from this study strongly suggest that a prospective application of a modified HEART score with POC troponin is warranted.

10. Gilbert EH, Lowenstein SR, Koziol-McLain J, Barta DC, Steiner J. Chart reviews in emergency medicine research: where are the methods? Ann Emerg Med. 1996; 27(3):305-308.

11. Mahler SA, Hiestand BC, Goff DC Jr., Hoekstra JW, Miller CD. Can the HEART score safely reduce stress testing and cardiac imaging in patients at low risk for major adverse cardiac events? Crit Pathw Cardiol. 2011;10(3):128-133.

12. Six AJ, Cullen L, Backus BE, et al. The HEART score for the assessment of patients with chest pain in the emergency department: a multinational validation study. Crit Pathw Cardiol. 2013;12(3):121-126.

13. Backus BE, Six AJ, Kelder JC, et al. A prospective validation of the HEART score for chest pain patients at the emergency department. Int J Cardiol. 2013;168(3): 2153-2158.

14. Thygesen K, Alpert JS, White HD, et al. Universal definition of myocardial infarction. Circulation. 2007;116(22):2634-2653.

15. Mahler SA, Riley RF, Hiestand BC, et al. The HEART Pathway randomized trial: identifying emergency department patients with acute chest pain for early discharge. Circ Cardiovasc Qual Outcomes. 2015;8(2):195-203.

16. Mahler SA, Riley RF, Russell GB, et al. Adherence to an accelerated diagnostic protocol for chest pain: secondary analysis of the HEART Pathway randomized trial. Acad Emerg Med. 2016;23(1):70-77.

17. Riley RF, Miller CD, Russell GB, et al. Cost analysis of the History, ECG, Age, Risk Factors, and initial Troponin (HEART) Pathway randomized control trial. Am J Emerg Med. 2017;35(1):77-81. 